\title{
Use of suction during endoscopic ultrasound-guided fine needle biopsy of solid pancreatic lesions with a Franseen-tip needle: a pilot comparative trial
}

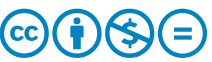

\author{
Authors \\ Lopes $^{3}$, Helena Barroca ${ }^{3}$, Guilherme Macedo ${ }^{1,2}$ \\ Institutions \\ 1 Gastroenterology Department, Centro Hospitalar e \\ Universitário São João, Porto, Portugal \\ 2 Faculty of Medicine, University of Porto, Portugal \\ 3 Pathology Department, Centro Hospitalar e \\ Universitário São João, Porto, Portugal
}

Pedro Costa-Moreira*, 1,2, Filipe Vilas-Boas*, 1,2, Diana Martins ${ }^{3}$, Pedro Moutinho-Ribeiro ${ }^{1,2}$, Susana Lopes ${ }^{1,2}$, Joanne

submitted 10.9.2020

accepted after revision 19.11 .2020

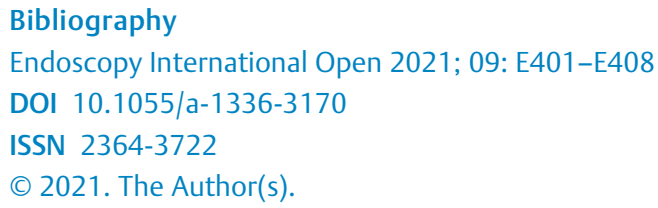
Commons Attribution-NonDerivative-NonCommercial License, permitting copying and reproduction so long as the original work is given appropriate credit. Contents may not be used for commecial purposes, or adapted, remixed, transformed or built upon. (https://creativecommons.org/licenses/by-nc-nd/4.0/)

Georg Thieme Verlag KG, Rüdigerstraße 14,

70469 Stuttgart, Germany

Corresponding author

Pedro Costa-Moreira, MD, Gastroenterology Department, Centro Hospitalar São João, Porto. Al. Prof. Hernâni Monteiro, 4200 - 319 Porto, Portugal

Fax: +351225513601

pedromoreira.med@gmail.com

Supplementary material is available under https://doi.org/10.1055/a-1336-3170

\section{ABSTRACT}

Background and study aims The utility of suction during endoscopic ultrasound (EUS) fine-needle biopsy (FNB) using Franseen-tip needle remains unclear and has not been evaluated in randomized trials. We designed a randomized crossover trial to compare the diagnostic yield during EUSFNB using a 22G Franseen-tip needle, with and without standard suction.

Patients and methods Consecutive patients undergoing EUS-guided sampling of solid pancreatic lesions were recruited. A minimum of two passes were performed for each case: one with $20-\mathrm{mL}$ syringe suction $\left(\mathrm{S}^{+}\right)$and another without (S-). The order of passes was randomized and the pathologist blinded. The endpoints were the diagnostic yield and the impact of blood contamination in the diagnosis.

Results Fifty consecutive patients were enrolled. The overall diagnostic accuracy was $84 \%$. A diagnosis of malignancy was obtained in 70 samples: 36 in the S+group and 34 in the S-group. A statistically significant difference was seen in the diagnostic accuracy (S+: $78 \%$ vs. $S-: 72 \%, P<0.01)$ and blood contamination (S+: $68 \%$; S-: $44 \%, P<0.01$ ). The sensitivity, specificity, negative likelihood ratio and positive likelihood ratio for S+vs. S-samples were $76.6 \%$ vs. $73.9 \%$, $100 \%$ vs. $100 \%$ and 0.23 vs. 0.26 , NA vs NA, respectively. A negative impact of blood contamination in the overall diagnostic yield wasn't seen, even in samples where suction was used (OR 0.36, $P=0.15$ )

Conclusions We found a higher diagnostic yield with the use of suction. It was associated with a higher degree of sample blood contamination that did not affect the diagnostic performance.

\section{Introduction}

Endoscopic ultrasound-guided tissue acquisition (EUS-TA) has become the procedure of choice for pathological diagnosis of solid pancreatic lesions [1].

\footnotetext{
* These authors contributed equally.
}

Since its introduction, EUS fine-needle aspiration (FNA) had been done with narrow-caliber needles. This technique, associated with the processing of the material obtained in smears, had some limitations, either depending on the poor cellular representation or by the hemorrhage conditioned by the technique, which limited the cytological diagnosis. To overcome the limitations associated with FNA, new needles (fine needle 
biopsy - FNB) with unique geometry of the cutting tip or a sideslot (core trap) at the distal needle portion were developed [2].

FNB needles are designed to preserve tissue architecture. The use of FNB associated with cell block sample processing instead of smearing, allows for not only histological observation but also conduct of immunohistochemistry studies with appropriate controls. It potentially eliminates the need for rapid onsite evaluation (ROSE), resulting in cost savings [3-5].

New EUS-FNB needles with innovative tip geometry (opposing bevel design), namely the Fork-tip (SharkCore, Medtronic, Minneapolis, Minnesota, United States) and Franseen-tip (Acquire, Boston Scientific Co., Marlborough, Massachusetts, United States) have been recently introduced. The Franseen design has a tip with three symmetric beveled cutting edges designed to get deep into the tissue and obtain sample tissue volume due to its large crown-tip area. Its structure with an electropolished tip improves the control and stability of the needle and allows penetrating the tissue, minimizing sample tearing and fragmentation [6]. Apart from the improved histological yield, both the Franseen-tip and the Fork-tip needle demonstrated excellent diagnostic accuracy (>90\%), with or without ROSE [6].

Previous studies of the EUS-FNA technique showed that standard suction (negative pressure, applied with a 10- or 20$\mathrm{mL}$ suction syringe connected to the needle) increases the rate of procuring diagnostic samples and the sample cellularity [79]. One of these studies [7] showed that $20-\mathrm{mL}$ suction is superior to $10-\mathrm{mL}$ suction. However, the application of suction often results in increased smear bloodiness, which can affect the cytological diagnostic interpretation [10].

The role of the different aspiration techniques when performing FNB was assumed from previous studies on FNA needles, despite the differences in the sample processing techniques (smear/liquid-based techniques for cytology generally used in FNA vs. tissue/cell block for histology in FNB) [3]. A recent randomized trial compared two sampling methods (standard suction or stylet slow-pull) during endoscopic ultrasoundguided FNB with a reverse-bevel needle in patients with pancreatic solid lesions. Meanwhile, the utility of suction in EUS-FNB with the new needles remains unclear and has not been evaluated in randomized controlled trials (RCTs). It remains uncertain whether the results from studies using FNA needles can be extrapolated to sampling with FNB needles [11].

We designed a randomized crossover trial to compare the diagnostic yield and quality of tissue specimen during EUS-FNB using a 22G Fransen-tip needle, with and without standard syringe suction.

\section{Patients and methods}

\section{Study design and patient population}

This was a prospective, randomized, crossover trial. Consecutive patients referred for EUS-TA of solid pancreatic lesions between June 2019 and March 2020 were included.

Written informed consent was obtained from all patients for the procedure. The study was carried out under the Declaration of Helsinki and was approved by the local Ethics Committee
("Comissão de Ética para a Saúde do Centro Hospitalar São João/Faculdade de Medicina da Universidade do Porto”). The study was registered in Clinical-Trials.gov (Identifier: NCT 04164017) and met the Standards for Reporting Diagnostic Accuracy Studies (STARD) and the Consolidated Standards of Reporting Trials (CONSORT) statements.

Adult patients ( $\geq 18$ years) with a diagnosis of pancreatic solid lesion based on prior cross-sectional imaging (performed within 6 months before randomization) were eligible. Patients were excluded at the time of EUS if the lesion was determined to be a cyst. Other exclusion criteria included uncorrectable coagulopathy (international normalized ratio $>1.5$ ) or thrombocytopenia (platelet $<50,000 / \mathrm{mm}^{3}$ ), the absence of fasting ( 2 hours without clear liquids and 6 hours without solid foods); clinical suspicion of upper digestive tract obstruction; an episode of acute pancreatitis within four weeks before EUS; respiratory failure; hemodynamic instability; pregnancy; or the refusal to participate in the study.

\section{EUS-FNB procedure}

All procedures were performed under deep sedation by three endosonographers (FVB, PMR, SL), each of them with experience in over 1000 cases. Curvilinear array echoendoscopes from Pentax and Olympus (GF-UCT180, Olympus, Pennsylvania, United States or EG-3870UTK, Pentax Medical, Tokyo, Japan) were used.

EUS-FNB was performed using one of the new special geometry tip needles (AcquireTM, Boston Scientific, Marlborough, Massachusetts, United States). A 22G needle was used in all cases

The intervention order was determined randomly using computer-generated randomization assignments that were placed in sealed envelopes and opened by the endoscopy nurse during the procedure.

After initial lesion characterization in B mode, EUS-TA was performed using fanning and at least ten back-and-forth movements inside the target lesion on every pass. When syringe suction was used, negative pressure was neutralized before needle withdrawing from the lesion, as per ESGE guidelines [11].

Head and uncinate lesions were punctured through the duodenum, while body and tail lesions were punctured via the stomach.

A minimum of two passes were performed for each case: one with $20 \mathrm{~mL}$ syringe air suction and another without suction (suction, S+; no suction, S-). The order of each pass was defined in the randomization process (order A: $\mathrm{S}+/ \mathrm{S}-$; order $\mathrm{B}$ : $S-/ S+)$. The fanning technique was used in all passes, according to ESGE guidelines [11]. Since the "slow-pull technique" applies some negative pressure in the needle ( $5 \%$ of the force generated with the syringe) $[12,13]$ in the cases without suction $(\mathrm{S}-$ ) the stylet was not used during the FNB puncture.

The specimen obtained in each pass was expelled using the needle stylet to a formalin container and sent to the Pathology Department (one formalin container for each pass - container A for the first pass; container B for the second pass). Macroscopic on-site evaluation (MOSE) by the endosonographer was performed based on the capacity to see a yellowish core of tis- 
sue in the formalin bottle.After this simples evaluation it was determined by the endoscopist the need for additional passes after the two defined by the protocol. If that was that case, the subsequent specimens were collected with the use of suction and sent in additional formalin containers.

All procedures were performed as day cases. Patients were observed in the endoscopy unit recovery area as per routine protocol until fully awake, being discharged if asymptomatic.

\section{Histologic assessment}

Histological processing in cell blocks were performed with HistoGelTM (Thermo Scientific, Waltham, Massachusetts, United States) and histological slides were stained with haematoxylineosin. Additional stains were performed at the discretion of the pathologist if further information for diagnosis was required. The pathologists were blinded to the order of passes.

The FNB samples were reported by two of three pancreatic pathologists. All samples were analysed in a weekly pathology meeting for pancreatic pathology cases. Every case was reported with a prose report describing the findings, with an interpretation and a final diagnostic category according to the the $\mathrm{Pa}$ panicolaou Society of Cytopathology guidelines [14].

Based on a previous study describing sampling techniques in FNA [8], we assessed "sample quality" through the number of diagnostic samples and bloodiness. This was evaluated by a questionnaire (filled by the pathologists) (Appendix 1) concerning the quality of the sample for each pass/formalin container. A diagnostic sample was defined as a set containing adequate cellular material for a conclusive histological diagnosis.

The bloodiness of each sample was evaluated and graded into four levels ( $\mathbf{F i g . 1}$ ), as previously reported in the study by Lee et al. [8]: 'none', absent blood cells; 'low', a few blood cells without affecting histological diagnosis; 'moderate', partially obscured by blood cells but possible histological diagnosis; 'high', hidden by blood cells leading to inadequate interpretation.

\section{Definitions and endpoints}

After the pathology report, the FNB samples were, for the purpose of statistic evaluation, categorized either as "non-diagnostic" or as "diagnostic" [14].

The primary endpoint was the diagnostic yield. Accuracy, sensitivity, and specificity, were determined for each sampling method using the final pathology diagnosis. The secondary outcome measures were the evaluation of specimen blood contamination, technical success and the occurrence of adverse events (AEs).

Adenocarcinoma, neuroendocrine tumors, pseudopapillary neoplasms and pancreatic metastases were defined as neoplastic/malignant lesions (true positive). The gold standard was defined as follows: for resectable cases, surgical histology was considered the gold standard, whereas for unresectable or benign cases, final pathology diagnosis with a compatible clinical outcome, at 6-month follow-up, was considered the gold standard. A negative pathology result was confirmed with clinical data and/or imaging at a 6-month follow-up. Sensitivity was

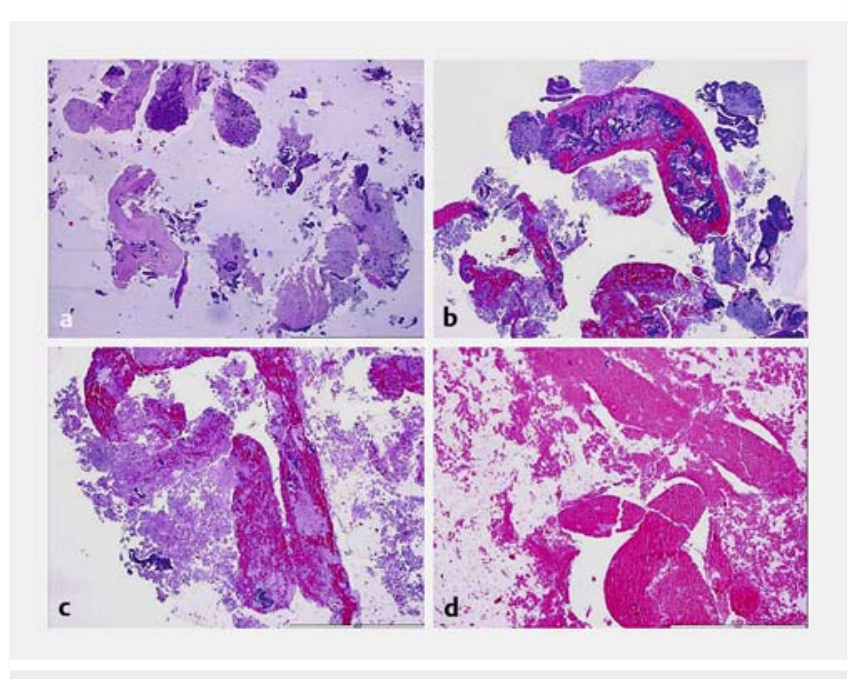

-Fig. 1 Grading of bloodiness in the histological samples of fineneedle biopsy (hematoxylin-eosin-stained slide, $40 \times$ ). a None absent blood cells. b Low - although cellular groups are involved in fibrin and blood, this does not affect the histological diagnosis. c Moderate - bleeding created by the process of biopsy makes it difficult for neoplastic cells to enter the needle. $\mathbf{d}$ High - although the histological diagnosis is not affected, the neoplastic representation is low, due to bleeding created by the biopsy. a, b, and c Adenocarcinoma - Papanicolau Category VI (Malignant). a, b Epithelial atypical cells forming duct-like structures, which infiltrate the pancreatic parenchyma and c elicit a strong desmoplastic stromal reaction or are detached. $\mathbf{d}$ Inadequate/insufficient sample (blood) - Papanicolau Category I (Non-diagnostic).

defined as the true positive rate using histological diagnosis as the gold standard.

Specificity was defined as the proportion of patients correctly identified on histology as having no malignancy concerning all patients without malignancy, as confirmed in comparison with the gold standard.

Technical success was defined as the ability to sample the target lesion with visualization of a core in the collecting bottle.

\section{Statistical analysis}

This was a pilot study aiming to obtain estimates of a sample size to design a larger one. We expected similar diagnostic performances of the two sampling methods. As a consequence, this study was designed as an equivalence study of alternative methods.

Continuous variables were reported as mean/median and interquartile range, and categorical variables were shown as frequency and percentage. Continuous variables were analyzed using Student's t-test. Sampling rate and diagnostic accuracy were compared with Fisher's exact test. All index procedures were included in the calculations without excluding technical failures or cases with poor yield (intention-to-diagnose analysis). No repeated EUS procedures were included in the calculation of the accuracy. In the statistical sense, all neuroendocrine tumors, irrespective of tumor grading (G1-G3) were considered malignant lesions. 


\begin{tabular}{|c|c|}
\hline \multicolumn{2}{|l|}{ Patient characteristic } \\
\hline - Male gender, n(\%) & $23(46 \%)$ \\
\hline - Age, mean $\pm S D$ (years) & $65.5 \pm 11.8$ \\
\hline \multicolumn{2}{|l|}{ EUS characteristic } \\
\hline \multicolumn{2}{|l|}{ Mass location } \\
\hline - Pancreatic body, n (\%) & $23(46 \%)$ \\
\hline - Pancreatic head, n (\%) & $19(38 \%)$ \\
\hline - Uncinate process, $\mathrm{n}(\%)$ & $5(10 \%)$ \\
\hline - Pancreatic tail, n (\%) & $3(6 \%)$ \\
\hline \multicolumn{2}{|l|}{ Lesion size } \\
\hline - Major diameter, mean \pm SD, mm & $34.8 \pm 13.1$ \\
\hline - Minor diameter, mean $\pm S D, m m$ & $28.4 \pm 10.9$ \\
\hline \multicolumn{2}{|l|}{ Limits and homogeneity of the lesion } \\
\hline - Regular limits, n (\%) & $6(12 \%)$ \\
\hline - Irregular limits, n (\%) & $44(88 \%)$ \\
\hline - Homogeneous lesion, n (\%) & $5(10 \%)$ \\
\hline - Heterogeneous lesion, n (\%) & $45(90 \%)$ \\
\hline - Cystic areas inside the solid lesion, $n$ (\%) & $15(30 \%)$ \\
\hline \multicolumn{2}{|l|}{ Echogenicity of lesion } \\
\hline - Hypoechogenic, n (\%) & $49(98 \%)$ \\
\hline - Hyperechogenic, n (\%) & $1(2 \%)$ \\
\hline - Vascular involvement, n (\%) & $20(40 \%)$ \\
\hline \multicolumn{2}{|l|}{ Real-time transient elastography } \\
\hline - Homogeneous blue pattern, n (\%) & $12(24 \%)$ \\
\hline - Heterogeneous blue pattern, n (\%) & $23(46 \%)$ \\
\hline - Green patterns, n (\%) & $0(0 \%)$ \\
\hline - Not described, n (\%) & $15(30 \%)$ \\
\hline Strain ratio, median (IQR) & $26.0(12.5-48.7)$ \\
\hline
\end{tabular}

Differences were considered statistically significant when $P$ $<0.05$. Statistical analyses were performed using SPSS version 24 for Windows (SPSS Inc., Chicago, Illinois, United States).

\section{Results}

\section{Patient demographics and tumor characteristics}

A total of 50 consecutive patients were enrolled over a 13month period. The mean age of the included subjects was $65.5 \pm 11.8$ years; $46 \%(n=23)$ were male. All included patients were randomized and underwent paired FNB sampling with and without syringe suction, as described above.

Baseline patient characteristics and EUS characterization of pancreatic lesions are presented in $>$ Table $\mathbf{1}$. The mean size of
- Table 2 Technical characteristics and histological results.

\section{FNB procedure}

Number of passes

- Two passes (“per protocol”), n(\%)

$36(72 \%)$

- Three passes, $\mathrm{n}(\%)$

$13(26 \%)$

- Four passes, $\mathrm{n}(\%)$

Site of FNB luminal puncture

- Gastric wall, $\mathrm{n}(\%)$ $26(52 \%)$

- Duodenal wall, n(\%) $24(48 \%)$

Histological diagnosis

Conclusive cases

- With the two passes "per protocol", n(\%) $42(84 \%)$

- All conclusive cases, $n(\%)$ $42(84 \%)$

Pathologic diagnosis

- Pancreatic adenocarcinoma, $\mathrm{n}(\%)$ $29(58 \%)$

- Neuroendocrine tumor, $\mathrm{n}(\%)$ $7(14 \%)$

- Metastasis of solid tumor, $\mathrm{n}(\%)$ $3(6 \%)$

- "Mass-forming" chronic pancreatitis, n(\%) $2(4 \%)$

- Solid pseudopapillary neoplasm $1(2 \%)$

- Inconclusive, $\mathrm{n}(\%)$ $8(16 \%)$

Final diagnosis

- Malignant cases (“true positive”), n(\%) $46(96 \%)$

- Benign cases (“true negative”), n(\%) $4(8 \%)$

Best sample (pathologist's subjective interpretation)

- Sample with suction (S+) $21(42 \%)$

- Sample without suction (S-) $21(42 \%)$

- No differences/not evaluable $8(15.4 \%)$

FNB, fine needle biopsy.

the lesion was $34.8 \pm 13.1 \mathrm{~mm}$. Lesions were located in the head/uncinate process $(n=24,48 \%)$, body $(n=23,46 \%)$, and pancreatic tail $(n=3 ; 6 \%)$. Real-time elastography was performed in 35 cases (70\%) and helped to choose the best puncture site within the lesion.

After the visual interpretation of the EUS findings, the suspected diagnosis of malignancy was made in the majority of cases ( $n=46,92 \%)$. Only in four cases the lesions were suspected to be benign (mass-forming chronic pancreatitis).

\section{Tissue acquisition techniques and diagnostic performance.}

The Franseen-tip needle $22 \mathrm{G}$ needle was used with technical success in all patients.

The technical aspects concerning the procedures are presented in > Table 2. All lesions were submitted to a minimum of two passes ("per protocol” analysis). In 14 cases (28\%), MOSE 
- Table 3 Comparison of diagnostic performance of the two techniques.

\begin{tabular}{|l|l|l|}
\hline & S+samples & S-samples \\
\hline True positive & 36 & 34 \\
\hline False positive & 0 & 0 \\
\hline True negative & 3 & 2 \\
\hline False negative & 11 & 14 \\
\hline Sensitivity & $76.6 \%$ & $73.9 \%$ \\
\hline Specificity & $100 \%$ & $100 \%$ \\
\hline Negative likelihood ratio & 0.23 & 0.26 \\
\hline Positive likelihood ratio & $\mathrm{NA}$ & $\mathrm{NA}$ \\
\hline Diagnostic yield & $78 \%$ & $72 \%$ \\
\hline
\end{tabular}

NA, not applicable; S+, sample with suction; S-, sample without suction.

by the endosonographer determined the need for additional passes (three passes in 13 cases, four passes in one case). According to lesion localization, the endoscope was located in the stomach in $52 \%(n=26)$ of the FNB procedures, and in the duodenum in the remaining cases $(n=24)$.

The overall diagnostic accuracy was $84 \%$ (42/50 cases). Per protocol analysis (based on the two mandatory passes) revealed a similar diagnostic yield.

The randomization resulted in an equitable distribution of the study intervention (order $\mathrm{A}[\mathrm{S}+/ \mathrm{S}-], \mathrm{n}=24$; order $\mathrm{B}[\mathrm{S}-/ \mathrm{S}+]$, $n=26)$. There were no differences in the diagnostic yield between the two study arms (order A: 21/24; order B: 21/26; $P=$ 0.52 ). The final pathological report contained the evaluation of the pathologist's perspective of the most informative/ representative sample for each case. There was no difference between S+and S- when the 'best sample' was considered respective to the order in study intervention $(S+: n=21,42 \%$; $S-: n=$ $21,42 \%$; not evaluable, $\mathrm{n}=8,16 \%, P=0.53)$. Taking into account only the neoplastic/malignant lesions, there was a similar proportion ( $S+, n=21,45.7 \%$; $S-, n=19,41.3 \%$; not evaluable, $\mathrm{n}=6,13 \% ; P=0.64)$.

A diagnosis of malignancy was obtained in 70 samples/bottles: 36 in the S+group and 34 in the S-group (> Table 3 ). In 5 samples/bottles ( 3 in the $S+$ group and 2 in the $S$-group) with benign histological findings at EUS-FNB, the definitive diagno- sis was chronic pancreatitis (considered true negative). There were samples with false negative results (11 in the $S+$ group and 14 in the $S$-group). Comparing the two groups (S+vs. $S-$ ), the sensitivity, specificity, negative likelihood ratio and positive likelihood ratio were $76.6 \%$ vs. $73.9 \%, 100 \%$ vs. $100 \%, 0.23$ vs. 0.26 and NA in both. The diagnostic accuracy of each group of samples was $78 \%$ for $\mathrm{S}+$ samples and $72 \%$ for $\mathrm{S}$-samples. Based on the statistical comparison of the diagnostic yield between the groups, a statistically significant difference was seen (6 cases with an inconclusive result in $\mathrm{S}$-samples that had a conclusive diagnosis in S+sample vs. 3 cases with an inconclusive result in $\mathrm{S}+$ samples that had a definitive diagnosis in $\mathrm{S}$-sample, $\mathrm{p}<0.01$ ) ( Table 4).

\section{Bloodiness of the samples}

For each sample in the protocol, the degree of blood contamination was evaluated using a grading score ( $\triangleright$ Fig. 1 and $\triangleright$ Table 5). The presence of blood contamination was seen in 34 cases $(68 \%)$ of the $S+$ samples (14 cases with moderate/high contamination) and 22 cases (44\%) of the S-samples ( 9 cases with moderate/high contamination) ( $>$ Table 6 ). High bloodiness was present in all non-diagnostic samples ( $\mathrm{S}+: 4$ cases in 11 non-diagnostic samples; $\mathrm{S}-\mathbf{3}$ cases in 14 non-diagnostic samples).

Comparing the two sampling techniques regarding the degree of blood contamination, we found a statistically significant difference between the groups ( $S+: n=32,68 \%$; $S-: n=22,44$ $\%, P<0.01)(\triangleright$ Table5). In a logistic binomial and univariate sub-analysis of the impact of any grade of bloodiness in the ability to provide a conclusive histological diagnosis, a negative impact in the overall diagnostic yield wasn't seen, even in samples where suction was used (S+: OR 1.88, CI95\% 0.48-7.36, $P$ $=0.37$ vs. S-: OR 0.71; CI95\% 0.21-2.47, P=0.05) ( Table 6).

Considering only the cases with moderate/high blood contamination, the prevalence of non-conclusive samples was significantly higher in $\mathrm{S}$-samples $(\mathrm{S}+: \mathrm{n}=5 / 14$ samples with moderate/high bloodiness vs. $6 / 36$ samples with none/low bloodiness, $P=0.05$; $S-: n=5 / 9$ samples with moderate/high bloodiness vs. 9/41 samples with none/low bloodiness, $P=0.04$ ). However, a negative impact in the overall diagnostic yield wasn't seen in the regression model ( $S+$ : OR 0.36, CI95\% $0.09-0.46, P=0.15$; S-: OR 0.23, CI95\% 0.05-1.0, $P=0.05$ )

- Table 4 Comparison of the diagnostic accuracy between the sampling techniques.

\begin{tabular}{|l|l|l|l|}
\hline & \multicolumn{2}{|c|}{ S-samples } & Inconclusive Result \\
\hline \multirow{2}{*}{ S+samples } & Conclusive result & $33(66 \%)$ & $6(12 \%)$ \\
\cline { 2 - 4 } & Inconclusive result & $3(6 \%)$ & $8(16 \%)$ \\
\hline & & $36(72 \%)$ & $14(28 \%)$ \\
\hline
\end{tabular}


- Table 5 Comparison of the bloodiness between the sampling techniques.

\begin{tabular}{|l|c|c|c|}
\hline Blood contamination & S+samples & S-samples & $P$ \\
\hline None, $\mathrm{n}(\%)$ & $16(32 \%)$ & $28(56 \%)$ & $<0.01{ }^{1}$ \\
\hline Low, $\mathrm{n}(\%)$ & $20(40 \%)$ & $13(26 \%)$ & \multirow{2}{*}{} \\
\hline Moderate, $\mathrm{n}(\%)$ & $10(20 \%)$ & $6(12 \%)$ & \\
\hline High, $\mathrm{n}(\%)$ & $4(8 \%)$ & $3(6 \%)$ & \\
\hline Total of samples, $\mathrm{n}(\%)$ & $50(100 \%)$ & $50(100 \%)$ & \\
\hline${ }^{1} \mathrm{X}^{2}$ test & & & \\
\hline
\end{tabular}

\section{Adverse events}

There were no procedure-related serious AEs (complications resulting in a physician visit or an episode of hospitalization or extension of an existing hospital stay, significant disability, or death). In one patient with a neuroendocrine tumor submitted to the two "per protocol" passes, self-limited bleeding was detected in the puncture site of the duodenal wall. Hemorrhagic relapse was not documented.

\section{Discussion}

In the present study, we found that the diagnostic accuracy of Franseen-tip 22G FNB needle was significantly different with the use of syringe suction ( $S+: 78 \%$ vs. $S-: 72 \%, P<0.01$ ). Standard syringe suction resulted in a higher degree of specimen blood contamination, that had no impact in the final diagnosis.

To the best of our knowledge, this is the first prospective, randomized trial to assess the impact of suction on the diagnostic yield of EUS-FNB of solid pancreatic lesions using a Franseentip $22 \mathrm{G}$ needle.

Concerning the use of suction during EUS-FNA of solid pancreatic lesions, two randomized trials using 22 and 25G FNA needles found that syringe suction improved sensitivity and accuracy for malignancy compared to no suction $[7,8]$
Previous studies reporting on the use of new tip geometry needles in the pancreas or other lesions were not controlled for the use of suction [15-18]. A recent multicenter retrospective study by Adler et al. [18] evaluated the performance of the Franseen-tip needle in 200 patients undergoing EUS-FNB with ROSE of different solid lesions (pancreas, adenopathy, subepithelial lesions and others). The technique for tissue acquisition, namely the use of suction and the performance of fanning, was left to the discretion of the endoscopist (the slow-pull technique was used in most procedures) and was not compared. As the authors state, the ideal method for operating FNB needles remains unknown. This study [18] showed that the tissue obtained by EUS-FNB was adequate for evaluation and diagnosis by ROSE in $98.5 \%$ of cases, and in $90 \%$ of cases, a visible tissue core was obtained.

In the case of the early reverse-bevel FNB needle, minimal suction provided by the slow-pull technique was compared with the standard syringe suction in 98 patients with solid pancreatic masses [19]. The authors found a higher but not statistically significant rate of diagnostic samples after a second pass using slow-pull than after a second pass (or even a first pass) using syringe suction. More recently, a prospective randomized multicenter trial compared the use of the slow-pull technique and standard syringe suction during FNB using an antegrade core trap needle (Procore forward-bevel 20G) in patients with solid pancreatic lesions [20]. The study showed that slow-pull and standard suction techniques are comparable in terms of blood contamination providing similar diagnostic sensitivity and accuracy.

To avoid a possible confusion of the negative pressure generated by the "slow-pull" technique, our study compared the application of aspiration with syringe vs. absence of any suction force. For this reason, stylet was not used during the puncture in all S-passes (it was only inserted in the needle to expel the specimen). Regarding the use of stylet, a recent study [21] was not able to establish a benefit of the aforementioned technique with respect to diagnostic accuracy, adequacy, quality, cellularity or blood contamination on EUS-FNB with a reverse-bevel needle.

- Table 6 Impact of the bloodiness on diagnostic accuracy between sampling techniques.

\begin{tabular}{|c|c|c|c|c|c|}
\hline & \multirow{2}{*}{$\begin{array}{l}\text { Sampling technique } \\
\text { Degree of contamination }\end{array}$} & \multicolumn{2}{|l|}{$S^{+}$} & \multicolumn{2}{|l|}{ S- } \\
\hline & & None or low & Moderate or high & None or low & Moderate or high \\
\hline \multirow[t]{3}{*}{ Histological diagnosis } & Conclusive & $30(60 \%)$ & $9(18 \%)$ & $32(64 \%)$ & $4(8 \%)$ \\
\hline & Inconclusive & $6(12 \%)$ & $5(10 \%)$ & $9(18 \%)$ & $5(10 \%)$ \\
\hline & $P^{1}$ & \multicolumn{2}{|l|}{0.05} & \multicolumn{2}{|l|}{0.04} \\
\hline \multirow[t]{4}{*}{ Impact of bloodiness } & Degree of contamination & Any degree & Moderate or high & Any degree & Moderate or high \\
\hline & OR & 1.88 & 0.36 & 0.71 & 0.23 \\
\hline & $\mathrm{Cl} 95 \%$ & $0.48-7.36$ & $0.09-0.46$ & $0.21-2.47$ & $0.05-1.0$ \\
\hline & $P^{2}$ & 0.37 & 0.15 & 0.05 & 0.05 \\
\hline
\end{tabular}


In our study, the performance of more passes in addition to the two determined by the protocol (performed in 14 cases by endoscopist decision) did not allow any additional diagnosis. MOSE was performed in a simplified form, only by the evaluation of the product in the formalin bottle (not in a glass slide 8 [22] or paper filter [23]), and without a measurement of the core length. These aspects can affect the MOSE accuracy in our study. However, Kaneko et al [24] recently evaluated the visible core cutoff lengths predictive of a correct diagnosis with a 22G Franseen needle. Similar to our method, in this study at least two needle passes were performed in all patients. The accuracy per pass was $92 \%$, and the authors showed that visible core lengths $>10 \mathrm{~mm}$ independently affected the correct diagnosis. Similar to these results, our study supports the recommendation of the European Society of Gastrointestinal Endoscopy (ESGE) [11] that suggests the performance of two to three passes when performing EUS-FNB without ROSE.

The overall diagnostic accuracy of the EUS-FNB (84\%) was similar to the previously reported target accuracy (> 85\%) [25]. The absence of ROSE could be a contributing factor explaining the presence of a small difference in the diagnostic accuracy in relation to previous studies. Notwithstanding, a recent metanalysis [6] showed that EUS-FNB using Franseen and fork-tip needles held similar results regardless the use of ROSE ( $95.9 \%$ without ROSE vs. $93.7 \%$ with ROSE, $P=0.25$ ).

The use of suction during EUS-TA has been associated with higher sample blood contamination [26]. The interference of blood with cytopathological reading is a well-known phenomenon in EUS-FNA [27]. The study by Lee et al. [8] that included 81 patients with solid pancreatic lesions that underwent EUSFNA demonstrated the presence of higher bloodiness in S+samples, as we found in our results. Concerning EUS-FNB using an antegrade core trap needle, Di Mitri et al. [20] on the other hand, found that the degree of blood contamination was not dependent on suction technique.

Despite the differences found in the degree of blood contamination between $\mathrm{S}+\mathrm{S}$-samples (similar to the previous studies involving FNA), our data showed that blood contamination didn't contributed to the decrease of diagnostic accuracy, even in samples where suction was used ( $S+$ : OR 1.88, $P=0.37$ ). Standard suction is known to increase the amount of obtained tissue [9]. In contrast with the traditional smears used for cytological examination, all samples in this study were processed for histological examination with cell block. This processing technique may overcome the negative impact of blood contamination usually seen in cytological smears [28]. Our results showed that despite an increase in the blood content in $\mathrm{S}+$ samples, the processing technique may overcome the impact in pathology interpretation in such samples.

The strengths of the present study include its prospective, randomized, crossover design and the blinding of the pathologists to the technical aspects of the tissue acquisition. In addition, the sample quality measurement included the quantitative analysis of the blood contamination and the subjective evaluation of the sample (with the classification of the best sample for diagnosis). The major weakness is related to the number of recruited patients, mainly due to the unicentric nature of this trial.

\section{Conclusion}

In conclusion, we found a higher diagnostic yield in EUS-FNB using a new Franseen-tip needle with the use of suction. The use of suction is associated with a higher degree of sample blood contamination that does not affect the diagnostic performance when in association with cell block sample processing. These results support the ESGE recommendation for the use of syringe suction for EUS-TA using FNA needles and new generation needles.

\section{Competing interests}

The authors declare that they have no conflict of interest.

\section{Clinical trial}

clinicaltrials.gov

NCT04164017

Clinical Trial Registration: Randomized, single-center, prospective crossover study with a single-blinded outcome assessor registered as NCT04164017 at clinicaltrials.gov

\section{References}

[1] Conti CB, Cereatti F, Grassia R. Endoscopic ultrasound-guided sampling of solid pancreatic masses: the fine needle aspiration or fine needle biopsy dilemma. Is the best needle yet to come? World J Gastrointest Endosc 2019; 11: 454-471

[2] Muniraj T, Aslanian HR. New developments in endoscopic ultrasound tissue acquisition. Gastrointest Endosc Clin N Am 2017; 27: 585-599

[3] Wong NACS. My approach to endoscopic ultrasound-guided fineneedle aspiration biopsy specimens of the pancreas. J Clin Pathol 2020; 73: 297-309

[4] Wani S, Muthusamy VR, McGrath CM et al. AGA White Paper: Optimizing Endoscopic Ultrasound-Guided Tissue Acquisition and Future Directions. Clin Gastroenterol Hepatol 2018; 16: 318-327

[5] Costa-Moreira P, Vilas-Boas F, Moutinho-Ribeiro P et al. Macroscopic on-site evaluation during EUS-fine needle biopsy with combined cyto and histological analysis may overcome the need of rapid on-site evaluation. Endosc Ultrasound 2019; 8: 432-433

[6] Mohan BP, Shakhatreh M, Garg R et al. Comparison of Franseen and fork-tip needles for EUS-guided fine-needle biopsy of solid mass lesions: A systematic review and meta-analysis. Endosc Ultrasound 2019; 8: 382-391

[7] Tarantino I, Di Mitri R, Fabbri C et al. Is diagnostic accuracy of fine needle aspiration on solid pancreatic lesions aspiration-related? A multicentre randomised trial Dig Liver Dis 2014; 46: 523-526

[8] Lee JK, Choi JH, Lee KH et al. A prospective, comparative trial to optimize sampling techniques in EUS-guided FNA of solid pancreatic masses. Gastrointest Endosc 2013; 77: 745-751

[9] Puri R, Vilmann P, Săftoiu A et al. Randomized controlled trial of endoscopic ultrasound-guided fine-needle sampling with or without suction for better cytological diagnosis. Scand J Gastroenterol 2009; 44: 499-504 
[10] Nakai Y, Isayama H, Chang K] et al. Slow Pull Versus Suction in Endoscopic Ultrasound-Guided Fine-Needle Aspiration of Pancreatic Solid Masses. Digestive Diseases and Sciences 2014; 59: 1578-1585

[11] Polkowski M, Jenssen C, Kaye P et al. Technical aspects of endoscopic ultrasound (EUS)-guided sampling in gastroenterology: European Society of Gastrointestinal Endoscopy (ESGE) Technical Guideline March 2017. Endoscopy 2017; 49: 989-1006

[12] Nakai Y, Isayama $\mathrm{H}$, Chang $\mathrm{KJ}$ et al. Slow pull versus suction in endoscopic ultrasound-guided fine-needle aspiration of pancreatic solid masses. Dig Dis Sci 2014; 59: 1578-1585

[13] Capurso G, Archibugi L, Petrone MC et al. Slow-pull compared to suction technique for EUS-guided sampling of pancreatic solid lesions: a meta-analysis of randomized controlled trials. Endosc Int Open 2020; 8: E636-E643

[14] Pitman MBL, Lester J. The Papanicolaou Society of Cytopathology System for Reporting Pancreaticobiliary Cytology. Switzerland: Springer International Publishing; 2015

[15] Bang JY, Hebert-Magee S, Hasan MK et al. Endoscopic ultrasonography-guided biopsy using a Franseen needle design: Initial assessment. Dig Endosc 2017; 29: 338-346

[16] El Chafic AH, Loren D, Siddiqui A et al. Comparison of FNA and fineneedle biopsy for EUS-guided sampling of suspected GI stromal tumors. Gastrointest Endosc 2017; 86: 510-515

[17] Nayar MK, Paranandi B, Dawwas MF et al. Comparison of the diagnostic performance of 2 core biopsy needles for EUS-guided tissue acquisition from solid pancreatic lesions. Gastrointest Endosc 2017; 85: 1017-1024

[18] Adler DG, Muthusamy VR, Ehrlich DS et al. A multicenter evaluation of a new EUS core biopsy needle: Experience in 200 patients. Endosc Ultrasound 2019; 8: 99-104

[19] El Haddad R, Barret M, Beuvon F et al. The slow-pull capillary technique increases the quality of endoscopic ultrasound fine needle biopsy samples in solid pancreatic lesions. Eur J Gastroenterol Hepatol 2016; 28: 911-916
[20] Di Mitri R, Mocciaro F, Antonini F et al. Stylet slow-pull vs. standard suction technique for endoscopic ultrasound-guided fine needle biopsy in pancreatic solid lesions using 20 Gauge Procore ${ }^{\mathrm{TM}}$ needle: $\mathrm{A}$ multicenter randomized trial. Dig Liver Dis 2020; 52: 178-184

[21] Yang M], Hwang JC, Yoo BM et al. A prospective randomized trial of EUS-guided tissue acquisition using a 25-gauge core biopsy needle with and without a stylet. Surg Endosc 2018; 32: 3777-3782

[22] Iwashita T, Yasuda I, Mukai T et al. Macroscopic on-site quality evaluation of biopsy specimens to improve the diagnostic accuracy during EUS-guided FNA using a 19-gauge needle for solid lesions: a singlecenter prospective pilot study (MOSE study). Gastrointest Endosc 2015; 81: 177-185

[23] Oh D, Seo DW, Hong SM et al. The impact of macroscopic on-site evaluation using filter paper in EUS-guided fine-needle biopsy. Endosc Ultrasound 2019; 8: 342-347

[24] Kaneko J, Ishiwatari H, Sasaki K et al. Macroscopic on-site evaluation of biopsy specimens for accurate pathological diagnosis during EUSguided fine needle biopsy using 22-G Franseen needle. Endosc Ultrasound 2020: doi:10.4103/eus.eus_49_20

[25] Domagk D, Oppong KW, Aabakken L et al. Performance measures for endoscopic retrograde cholangiopancreatography and endoscopic ultrasound: A European Society of Gastrointestinal Endoscopy (ESGE) Quality Improvement Initiative. United European Gastroenterol ] 2018; 6: 1448-1460

[26] Wallace MB, Kennedy T, Durkalski V et al. Randomized controlled trial of EUS-guided fine needle aspiration techniques for the detection of malignant lymphadenopathy. Gastrointest Endosc 2001; 54: 441447

[27] Jhala NC, Jhala DN, Chhieng DC et al. Endoscopic ultrasound-guided fine-needle aspiration. A cytopathologist's perspective. Am J Clin Pathol 2003; 120: 351-367

[28] Wong N. My approach to endoscopic ultrasound-guided fine-needle aspiration biopsy specimens of the pancreas. J Clin Pathol 2020; 73: 297-309 